\title{
The Role of the Supplementary Motor Area in Inhibitory Control in Monkeys and Humans
}

\author{
Claire Wardak \\ Centre de Neuroscience Cognitive, Unité Mixte de Recherche 5229, Centre National de la Recherche Scientifique-Université Claude Bernard Lyon 1, 69675 \\ Bron Cedex, France \\ Review of Chen et al.
}

Self-control relies on the ability to override inappropriate impulses or responses to external stimuli. Most studies of this ability have focused on reactive stopping, i.e., on inhibitory processes triggered reactively by an external signal. Yet recent developments in the cognitive neuroscience of inhibitory control have highlighted the role of another kind of inhibitory control: proactive mechanisms (for review, see Aron, 2010). Reactive and proactive inhibition can be distinguished from each other by how they are initiated. Reactive inhibition is always triggered by an external cue, such as a stop light, and is transient following this event. Proactive inhibition, in contrast, is a preparatory mechanism guided by internal processes and prevents a response to a subsequent event. It is guided endogenously, although not necessarily consciously, and can be sustained over many trials. It allows subjects (humans or animals) to modulate their responsiveness to anticipated stimuli by appropriately gating automatic motor responses according to the context (Jaffard et al., 2007).

Received Jan. 2, 2011; revised Feb. 1, 2011; accepted Feb. 1, 2011.

This work was supported by the Fondation de France. I thank P. Boulinguez, S. Ben Hamed, and K. Reilly for comments on the manuscript.

Correspondence should be addressed to Claire Wardak, Centre de Neuroscience Cognitive, Unité Mixte de Recherche 5229, Centre National de la Recherche Scientifique-Université Claude Bernard Lyon 1, 67 Boulevard Pinel, 69675 Bron Cedex, France. E-mail: wardak@isc.cnrs.fr.

DOI:10.1523/JNEUROSCI.0006-11.2011

Copyright $\odot 2011$ the authors $\quad 0270-6474 / 11 / 315181-03 \$ 15.00 / 0$
Several studies in humans have focused on identifying the cerebral network involved in inhibitory control, primarily using reactive control paradigms. These studies demonstrated the involvement of several frontal regions, including supplementary motor area (SMA) amd preSMA, as well as basal ganglia (for review, see Aron, 2010). It is unclear, however, whether these same regions are involved in proactive control mechanisms.

In previous studies, response inhibition was consistently found to involve pre-SMA, and the only evidence for the involvement of SMA came from one microlesion study in humans (Sumner et al., 2007). Therefore, Chen et al. (2010) studied the role of SMA in monkeys, in both reactive and proactive control of hand movements. They recorded local field potentials (LFPs), as well as single-unit and multiunit activity within the SMA of two monkeys performing a stop-signal task. The animals were trained to use a handlebar to move a cursor toward a target box that appeared in either of two possible locations on a screen. In some trials, a stop signal appeared and to be rewarded the monkey had to cancel (i.e., not move its hand) or correct (i.e., place the cursor back in the center if it had not yet reached the target) the cursor's movement it. The timing between the signal instructing the movement and the stop signal was varied, and the proportion of trials on which the monkeys were able to cancel their movement varied accordingly.
During this task, which studied reactive control to the presentation of the stop signal, a significant increase of power in the LFPs was associated with movement cancellation. This result suggests that SMA has a role in the reactive control of movement, confirming the results of a previous study that showed that some single neurons in the SMA influence movement cancellation (Scangos and Stuphorn, 2010).

The monkeys' behavior was also influenced by recent trial history; if the reaction time (in response to the presentation of the target) on one no-stop trial was fast, then the movement tended not to be cancelled on the next stop trial, whereas if the reaction time on a trial was slow, the movement was successfully cancelled on the next stop trial. Moreover, both monkeys adapted their strategies following stop trials, notably by slowing their reaction times after a noncancelled trial. These behavioral effects show that the level of motor readiness is adjusted according to previous trial history, thus reflecting a proactive control of movement. Interestingly, Chen and colleagues (2010) show that the changes in multiunit activity (significant increase of activity before the initiation of movement) and in LFPs (decrease of power in a low-frequency band and increase in a high-frequency band) correlated with the timing of the monkeys' movements. This neural activity, which started before the presen- 
tation of the target for the LFPs (i.e., before the monkey could plan its arm movement), was observed earlier in trials with fast reaction times. Moreover, the amplitude of SMA activity was modulated by trial history. These results strongly suggest the SMA is not only involved in reactive inhibitory control of movement, but is also important in proactive inhibition.

The involvement of the SMA in both reactive and proactive inhibitory control of movement in monkeys is in line with results recently obtained in humans that directly pinpoint the role of SMA in these two inhibitory control mechanisms. In humans, the role of SMA in proactive inhibitory control is difficult to evidence using standard neuroimaging techniques because it is temporally coupled, i.e., potentially confounded, with processes related to movement preparation. A group of recent fMRI experiments identified the network of areas involved in proactive control of movements by analyzing the mechanisms underlying suppression of movements that are represented in the motor system but not performed. These representations were created through motor imagery (Kasess et al., 2008) or by observing the actions of others (Dinomais et al., 2009). Kasess et al. (2008) studied the effective connectivity between the SMA and the primary motor cortex M1 during movement execution and mental imagery. They showed that lack of activation in M1 during imagery is caused by suppression from the SMA. Importantly, during motor execution, they found that SMA activation was sustained for several seconds throughout the readiness period before movement. Dinomais et al. (2009) compared the passive observation of movements and the active execution of the same movements and found that several regions, including the SMA, were activated in the passive condition (i.e., when execution had to be suppressed). These results complement those of Jaffard et al. (2008), who showed that several areas, including the SMA, are involved in a task requiring proactive inhibition. Behavioral evidence of this proactive control is observed on reaction times: intermixing target stimuli with nontarget stimuli dramatically increases reaction times to targets when compared with a control condition in which only target stimuli are presented within a block of trials, i.e., a condition that does not require the subject to stop themselves from reacting automatically to the next event. These findings suggest that the role of the SMA in the control of action is not only related to motor preparation, but also to proactive inhibitory control of movement initiation.

More recent studies highlight another potential role of SMA in inhibitory processes related to reactive control. Following the initial human microlesion study of Sumner et al. (2007), Boy et al. (2010a) found that automatic inhibitory mechanisms that suppress automatic motor activations evoked by cues are supported by GABA concentration in the SMA, as measured with magnetic resonance spectroscopy. The authors further support this conclusion in a companion fMRI study (Boy et al., 2010b) that shows that SMA, but not pre-SMA, signals are modulated in this inhibitory task.

Some of these human studies, in addition to indicating involvement of the SMA in inhibitory mechanisms, also suggest that the pre-SMA is not involved in some reactive (Sumner et al., 2007; Boy et al., 2010b) and proactive (Jaffard et al., 2008) contexts. The question of the differential involvement of these two regions in the monkey still remains to be addressed.

In a previous paper, Scangos and Stuphorn (2010) analyzed single-neuron data obtained by Chen et al. (2010), including data from both SMA and preSMA, and they failed to observe a difference between the two regions in reactive inhibitory control. In light of the data presented by Chen et al. (2010), the comparison of the multiunit activity and LFPs recorded in the two areas is expected to be much more informative about the specific roles of each area than spike analysis. Although Chen et al. (2010) recorded from both SMA and pre-SMA, they focused only on SMA in their paper, probably because they obtained an enormous amount of data. Some of the results presented are explicitly reported as not significantly different from those obtained from pre-SMA recordings, but it is not clear whether this also applies to their other observations.

Because the SMA and pre-SMA are connected, the exact functional role of the SMA in reactive and proactive inhibitory control remains unclear and not clearly differentiated from the role of the pre-SMA. For example, in Chen et al. (2010), some of the SMA recording patterns could have been influenced by signals from the pre-SMA. The significant changes in LFP power were mainly observed in two frequency bands: a high-frequency band $(60-150 \mathrm{~Hz})$, assumed to reflect the spiking activity of the region recorded from; and a lowfrequency band $(25-40 \mathrm{~Hz})$, thought to reflect intra-areal synaptic activity. This latter frequency band was significantly modulated at the earliest timing, before target presentation. Because LFPs reflect both incoming signals and local computations, this finding raises the question of the exact role of the SMA in the proactive control of the movement; the proactive signal might originate in another area projecting to the SMA (e.g., the pre-SMA) and thereby influence its neuronal activity.

An easy way to address the question of the precise roles of both the SMA and the pre-SMA and to distinguish their functional specificities in proactive and reactive inhibitory mechanisms is to use a perturbation technique (e.g., inactivation or microstimulation). Although recordings help elucidate the local computations at work and their precise temporal organization, the results they provide rely on correlations. Perturbation techniques provide causality: we perturb the normal functioning of a region and observe the behavioral consequences.

In conclusion, Chen et al. (2010) showed a tight correlation between the neuronal activity at different levels (single-unit, multiunit, and LFPs) within the SMA and both the proactive and reactive control of arm movements in monkeys. These data complement several lines of evidence suggesting the functional involvement of the SMA in both forms of inhibitory control in humans. We can thus plausibly suppose that the mechanisms at the neuronal level are the same in both species. The next step in understanding precisely how these multiple inhibitory mechanisms are implemented would be to try to dissociate the functional involvement of the SMA and pre-SMA in the monkey on the basis of the seminal studies in humans.

\section{References}

Aron AR (2010) From reactive to proactive and selective control: developing a richer model for stopping inappropriate responses. Biol Psychiatry. Advance online publication. Retrieved November 16, 2010. doi:10.1016/ j.biopsych. 2010.07.024.

Boy F, Evans CJ, Edden RA, Singh KD, Husain M, Sumner P (2010a) Individual differences in subconscious motor control predicted by GABA concentration in SMA. Curr Biol 20:1779-1785.

Boy F, Husain M, Singh KD, Sumner P (2010b) Supplementary motor area activations in un- 
conscious inhibition of voluntary action. Exp Brain Res 206:441-448.

Chen X, Scangos KW, Stuphorn V (2010) Supplementary motor area exerts proactive and reactive control of arm movements. J Neurosci 30:14657-14675.

Dinomais M, Minassian AT, Tuilier T, Delion M, Wilke M, N'Guyen S, Richard I, Aubé C, Menei P (2009) Functional MRI comparison of passive and active movement: possible inhibitory role of supplementary motor area. Neuroreport 20:1351-1355.
Jaffard M, Benraiss A, Longcamp M, Velay JL, Boulinguez P (2007) Cueing method biases in visual detection studies. Brain Res 1179:106-118.

Jaffard M, Longcamp M, Velay JL, Anton JL, Roth M, Nazarian B, Boulinguez P (2008) Proactive inhibitory control of movement assessed by event-related fMRI. Neuroimage 42:1196-1206.

Kasess CH, Windischberger C, Cunnington R, Lanzenberger R, Pezawas L, Moser E (2008) The suppressive influence of SMA on M1 in motor imagery revealed by fMRI and dynamic causal modeling. Neuroimage 40:828-837.

Scangos KW, Stuphorn V (2010) Medial frontal cortex motivates but does not control movement initiation in the countermanding task. J Neurosci 30:1968-1982.

Sumner P, Nachev P, Morris P, Peters AM, Jackson SR, Kennard C, Husain M (2007) Human medial frontal cortex mediates unconscious inhibition of voluntary action. Neuron 54:697-711. 